www.nature.com/jhg

\title{
Are GJB2 mutations an aggravating factor in the phenotypic expression of mitochondrial non-syndromic deafness?
}

\begin{abstract}
Haris Kokotas $^{1}$, Maria Grigoriadou ${ }^{1}$, George S Korres ${ }^{1}$, Elisabeth Ferekidou ${ }^{1}$, Aglaia Giannoulia-Karantana ${ }^{2}$, Dimitrios Kandiloros ${ }^{3}$, Stavros Korres ${ }^{3}$ and Michael B Petersen ${ }^{1}$

Hearing impairment is a frequent condition, and genes have an important role in its etiology. The majority of hearing loss occurs in non-syndromic form, with deafness being the only clinically recognizable feature. More than 60 nuclear genes or loci have been shown to be involved in non-syndromic hearing loss, but mutations in mitochondrial DNA also cause hearing impairment. Mitochondrial DNA mutations usually lead to progressive hearing loss with an age of onset varying from childhood to early adulthood. It is interesting to note that there is a great variability among phenotypes between individuals harboring the same mitochondrial mutation, even within the same family, and the phenotype may range from profound deafness to completely normal hearing. In the past years, the debate on mitochondrial mutations has been about the penetrance, the tissue specificity and the mechanisms of modifier genes that can modulate the severity of the phenotypic expression of the deafness-associated mitochondrial DNA mutations. Here we summarize evidence regarding modifying genes, and we discuss the effect of the coexistence of mitochondrial and GJB2 mutations in families reported to date.
\end{abstract}

Journal of Human Genetics (2010) 55, 265-269; doi:10.1038/jhg.2010.23; published online 19 March 2010

Keywords: aggravating factor; A1555G; deafness; GJB2; G7444A; mitochondrial; modifier gene; non-syndromic

\section{INTRODUCTION}

Hearing impairment is one of the most common sensory handicaps with a frequency of at least 1 out of 1000 at birth, whereas at the age of 80 years more than $50 \%$ of the elderly have developed hearing loss severe enough to impair communication. ${ }^{1}$ Mitochondrial DNA (mtDNA) mutations are present in $<1 \%$ of the children with prelingual deafness, ${ }^{2}$ but are more frequent at a later age. In the Caucasian population at least $5 \%$ of postlingual, non-syndromic hearing impairment is due to known mtDNA mutations, representing the most frequent cause of hearing loss after the 35delG mutation in the GJB2 gene encoding connexin $26(\mathrm{Cx} 26){ }^{3}$ Especially the mitochondrial genes MTRNR1 and MTTS1, encoding 12S rRNA and tRNA $^{\mathrm{Ser}(\mathrm{UCN})}$ respectively, have been found to be associated with non-syndromic hearing loss.

mtDNA is transmitted only through the maternal/matrilineal lineage. ${ }^{4}$ mtDNA mutations occur spontaneously at a high rate and most changes are neutral polymorphisms without clinical significance. ${ }^{5}$ Furthermore, there appears to be a class of slightly deleterious mutations that modify the risks of mitochondrial disease, as in a multifactorial model, and distinct mtDNA mutations may act synergistically to modulate disease expression. ${ }^{6,7}$ Consequently, it might be difficult to determine the pathogenicity of a new mtDNA variation. Mitochondrial disease is characterized by an impressive degree of variation, and both inter- and even intrafamilial variation is the rule rather than the exception. mtDNA mutations might both lead to syndromes, but also to very specific phenotypes limited to one organ, such as Leber's hereditary optic neuropathy and non-syndromic deafness. ${ }^{8}$ The existence of heteroplasmy might explain part of this variety. It is proposed that the accumulation of mtDNA mutations and the subsequent cytoplasmic segregation of these mutations during life is an important contributor both to the aging process and to several human degenerative diseases. ${ }^{9}$ Environmental factors (diet, toxic factors and so on) might further contribute to the progressive breakdown of mitochondrial function with age, resulting in a late-onset disease. According to the 'vicious cycle theory, ${ }^{10}$ somatic mtDNA mutations may accumulate with age as each of them may lead to further defects in mitochondrial function generating additional mtDNA mutations. This makes mitochondrial disease a paradigm for multifactorial inheritance.

Several mtDNA mutations in the MTRNR1 gene encoding the $12 \mathrm{~S}$ rRNA and the MTTS1 gene encoding the tRNA ${ }^{\mathrm{Ser}(\mathrm{UCN})}$ have been associated with non-syndromic deafness. ${ }^{8}$ The MTRNR1 C1494T and

${ }^{1}$ Department of Genetics, Institute of Child Health, 'Aghia Sophia' Children's Hospital, Athens, Greece; ²Department of Pediatrics, Athens University Medical School, 'Aghia Sophia' Children's Hospital, Athens, Greece and 'Department of Otorhinolaryngology—Head and Neck Surgery, Athens University Medical School, Hippokration Hospital, Athens, Greece

Correspondence: H Kokotas, Department of Genetics, Institute of Child Health, 'Aghia Sophia' Children's Hospital, Athens 11527, Greece.

E-mail: hkokotas@yahoo.gr

Received 8 January 2010; revised 21 February 2010; accepted 25 February 2010; published online 19 March 2010 
A1555G mutations have been associated with both aminoglycoside induced and non-syndromic hearing loss in many families worldwide. $^{8,11-15}$ The tRNA ${ }^{\mathrm{Ser}(\mathrm{UCN})} / \mathrm{CO} 1 \mathrm{G} 7444 \mathrm{~A}$ variant has been reported alone or in cosegregation with the A1555G or C1494T deafnesscausing mtDNA mutations. Although there is an ongoing debate regarding the pathogenicity of the G7444A variant, it has been reported in cases with non-syndromic hearing loss. ${ }^{16-24, \text { unpublished data }}$

The GJB2 nuclear gene maps on chromosome 13q11-q12 and encodes the gap junction protein Cx26. Loss or malfunction of gap junctions, as resulting from mutations in the GJB2 gene, may disrupt potassium movement from the hair cells through the supporting cell network back to the endolymph, leading to hearing impairment. Deafness can also be caused by defective permeation of inositol1,4,5-triphosphate through gap junctions. ${ }^{25} \mathrm{~A}$ limited number of GJB2 mutations lead to autosomal dominant hearing loss, and others lead to syndromic hearing loss with skin disorders as the accompanying symptom, such as palmoplantar keratoderma with hearing loss ${ }^{26}$ and keratitis-ichthyosis-deafness syndrome. ${ }^{27}$ In total, around 90 different GJB2 mutations have so far been reported to be associated with recessive, non-syndromic hearing loss. ${ }^{28}$ One specific mutation, 35delG, consists of deletion of a guanine $(G)$ in a stretch of six Gs. The relative contribution of the GJB2 gene to non-syndromic, prelingual deafness varies from 0 to $40 \%$ in the populations studied, ${ }^{29}$ demonstrating genetic heterogeneity. The degree of hearing impairment in 35delG homozygotes is in most cases severe or profound, but can also be mild or moderate. The hearing loss is usually prelingual and non-progressive, but some cases with progression have been reported. ${ }^{30-32}$

\section{COEXISTENCE OF GJB2 AND mIDNA DEAFNESS-CAUSING MUTATIONS}

In general, mtDNA mutations appear to be rare. The A1555G seems to be frequent in Spanish and Asian populations but is rare in other studied populations. ${ }^{11,14,16,33,34}$ Taking this into consideration, we can conclude that the possibility of detecting individuals which harbor both a deafness-causing mtDNA mutation and a mutation in the GJB2 gene is rather low. Logically, we expect to detect such individuals in populations with a high frequency of mtDNA or GJB2 mutations, or both. To our knowledge, only a few families harboring GJB2 and mtDNA mutations have been reported. The families originated from Spain, ${ }^{17}$ Japan $^{34}$ and Greece ${ }^{35, \text { unpublished data (Table 1). Several }}$ families have been reported to have one mtDNA deafness-causing mutation and several other mtDNA variants described as polymorphisms, but only a few individuals were found with more than one mtDNA deafness-causing mutation. ${ }^{16,19,22}$

\section{THE GJB2 35deIG AND A1555G mtDNA MUTATIONS IN A SPANISH FAMILY}

López-Bigas et al. ${ }^{17}$ studied the GJB2 35delG mutation in 154 deaf individuals with the A1555G mutation and also analyzed the entire coding region of GJB2 in 42 unrelated patients from their cohort. They detected only one family with the 35delG mutation in heterozygosity, including four patients also having the A1555G mutation and two patients with the A1555G alone. ${ }^{17}$ None of these individuals was treated with aminoglycosides. All four patients with the A1555G and the 35delG mutation had moderate hearing loss and the age of onset was $16-30$ years (I-IV, Table 1 ). The two family members with the A1555G but not the 35delG mutation also presented with moderate postlingual hearing impairment (V and VI, Table 1). No other changes in the coding region of the GJB2 gene were detected in this sample of 42 unrelated patients with the A1555G mtDNA mutation. ${ }^{17}$

\section{COEXISTENCE OF THE GJB2 235deIC AND THE A1555G mtDNA MUTATIONS IN JAPAN}

In their study, Abe et al. ${ }^{34}$ reported a high prevalence of GJB2 heterozygous mutations in 23 Japanese families bearing the A1555G mtDNA mutation. In total 8 of the 23 families were heterozygous either for 235delC, 299-300delAT, 176-191del, Y136X or V37I. They described a family in which potential interaction between GJB2 and a mitochondrial gene appeared to be the cause of hearing impairment. In that family, patients who were heterozygous for the GJB2 mutant allele showed hearing loss more severe than that seen in siblings lacking a mutant GJB2 allele, suggesting that heterozygous GJB2 alleles may synergistically cause hearing loss in the presence of an A1555G mutation. Two siblings had the A1555G mutation, aminoglycoside exposure and hearing loss (VII and VIII, Table 1). Their sister had two genetic factors, the GJB2 235delC mutation in heterozygosity and the A1555G mtDNA mutation, in addition to aminoglycoside exposure. She presented with profound deafness (IX, Table 1). They suggested that GJB2 mutations may sometimes be an aggravating factor in addition to aminoglycosides in the phenotypic expression of non-syndromic hearing loss associated with the A1555G mtDNA mutation. $^{34}$

\section{TWO GREEK FAMILIES HARBORING THE GJB2 35deIG AND A1555G mtDNA MUTATIONS}

We have previously reported our findings from the first large screening of the Greek population for the A1555G mtDNA mutation and described two families with the mutation. ${ }^{35}$ Both families had a history of aminoglycoside exposure but only one family also had the 35delG GJB2 mutation. In this family, the proband had a prelingual moderate hearing loss and was heterozygous for the GJB2 35delG mutation (X, Table 1). The coding region of the GJB2 gene in this case was analyzed by direct genomic sequencing to investigate the possible existence of a second mutation in compound heterozygosity with the 35delG mutation but no other mutation was found. His brother, with the A1555G mutation, was also a carrier of the 35delG GJB2 mutation and although his clinical history reported no use of aminoglycosides, he presented with a speech delay and sensorineural hearing loss of moderate degree (XI, Table 1). The A1555G mutation was verified in the maternal grandmother and in the mother, who also reported aminoglycoside exposure but had normal levels of hearing. The mother was negative for the GJB2 35delG mutation. ${ }^{35}$

In the other Greek family with the A1555G but not the $35 \mathrm{delG}$ mutation, the proband, which was also exposed to aminoglycosides, had profound deafness (XII, Table 1). However, his three male siblings and mother, also with the A1555G mutation but not exposed to aminoglycosides, had normal hearing. ${ }^{35}$

\section{A GREEK FAMILY WITH THE GJB2 35delG AND G7444A mIDNA MUTATIONS}

We recently identified a Greek family (unpublished data) harboring the rare G7444A mtDNA variant, previously associated with nonsyndromic hearing loss. ${ }^{16-24}$ Two siblings and their mother with the G7444A mtDNA mutation were heterozygous for the GJB2 35delG mutation. Although the proband presented with mild/moderate hearing impairment (XIII, Table 1), his brother and mother had normal levels of hearing.

\section{COEXISTENCE OF THE A1555G AND G7444A mtDNA MUTATIONS}

The coexistence of one mtDNA deafness-causing mutation and other mtDNA variants of unknown significance or the presence of two 
Table 1 Families harboring GJB2 and mtDNA deafness-causing mutations

\begin{tabular}{|c|c|c|c|c|c|c|c|c|}
\hline Origin of family (reference) & $\begin{array}{l}\text { Affected } \\
\text { (n) }\end{array}$ & Subject/gender & $\begin{array}{l}m t D N A \\
\text { mutation }\end{array}$ & $\begin{array}{l}\text { GJB2 } \\
\text { mutation }\end{array}$ & $\begin{array}{l}\text { Degree of } \\
\text { deafness }\end{array}$ & $\begin{array}{l}\text { Type of } \\
\text { deafness }\end{array}$ & $\begin{array}{l}\text { Age of onset } \\
\text { (years) }\end{array}$ & Aminoglycosides \\
\hline \multirow[t]{2}{*}{ Spain $^{17}$} & 4 & $(\mathrm{I}-\mathrm{IV}) ?$ & & 35 delG/- & Moderate & Postlingual & $16-30$ & No \\
\hline & & $(\mathrm{VI}) ?$ & & $-1-$ & Moderate & & $\sim 20$ & \\
\hline \multirow[t]{2}{*}{$J_{a p a n}^{34}$} & 3 & (VII) female & & $-1-$ & Severe? & Postlingual & $>9$ & Yes \\
\hline & & (VIII) female & A1555G & $-1-$ & Severe? & & $<5$ & \\
\hline Greece $^{35}$ & & (XI) male & A1555G & 35delG/- & Moderate & Postlingual & $<4$ & No \\
\hline Greece $^{35}$ & 1 & (XII) male & A1555G & $-1-$ & Profound & Postlingual & 2 & Yes \\
\hline Greece (unpublished data) & 1 & (XIII) male & G7444A & 35delG/- & Moderate & Postlingual & 3 & No \\
\hline
\end{tabular}

The proband of the second family from Greece, with the A1555G but not the 35delG GJB2 mutation presented with profound deafness, in contrast to the proband of the first Greek family who was also subjected to aminoglycosides, and the proband of the third Greek family with the G7444A and 35delG mutations.

mtDNA mutations in the same individual has also been shown. Pandya et al., ${ }^{16}$ demonstrated the coexistence of the A1555G and the G7444A mutations of the tRNA ${ }^{\text {Ser(UCN) }}$ gene in Mongolian deaf students. They suggested that patients with both mutations in the mitochondrial genome may present earlier onset and an increased severity of hearing loss than others with the A1555G mutation alone. They proposed digenic epistasis for A1555G and G7444A, especially in the absence of aminoglycoside exposure. ${ }^{16}$ Yuan et al. ${ }^{19}$ also reported coexistence of the A1555G and the G7444A mtDNA mutations in a Chinese family. They concluded that the G7444A mutation may lead to a defect in the processing of the L-strand RNA precursor, thus influencing the phenotypic expression of the A1555G mutation. However, López-Bigas et al. ${ }^{17}$ reported that the G7444A mutation was absent in 42 Spanish families harboring the A1555G mutation, and concluded that G7444A is unlikely to be a modifier of the wide clinical variability of the deafness phenotype because of the A1555G mutation in Spanish patients. ${ }^{17}$

\section{DISCUSSION}

The phenotypic variability between individuals harboring the same mtDNA mutation, even among persons within the same family, has been an interesting topic in the research field of non-syndromic deafness. The phenotype may range from profound deafness to complete normal hearing in families of individuals with homoplasmic or near-homoplasmic mtDNA mutations. Several factors can modulate phenotypic expression. The administration of aminoglycosides, as a triggering event in the case of the $\mathrm{A} 1555 \mathrm{G}$ mutation, is the prime example. ${ }^{36}$ The molecular mechanism responsible for aminoglycosideinduced ototoxicity remains controversial. Aminoglycoside-induced loss of auditory function is characterized by the gradual destruction of sensory hair cells in the cochlea, starting in the basal turns and ascending to the apex. ${ }^{37,38}$ Aminoglycosides have been reported to affect DNA, RNA and protein synthesis, and have an effect on metabolism. It has been hypothesized that aminoglycosides may form cochleotoxic metabolites, because saturating concentrations of aminoglycosides are present in the inner ear within hours after parenteral administration, although loss of auditory function develops gradually. ${ }^{39}$ Nonetheless, these mechanisms cannot adequately explain the selective vulnerability of hair cells, particularly as other cells and tissues of the cochlea can take up aminoglycosides without permanent toxic effects. ${ }^{40}$

Recently, studies on mitochondrial haplotypes have shown that certain haplogroups increase the penetrance of mtDNA deafness- causing mutations among different populations. ${ }^{41}$ For example, the haplogroup $\mathrm{B}$ increases the penetrance of hearing loss in Chinese subjects carrying the A1555G mutation, whereas the haplogroup B4 may increase the penetrance of hearing impairment associated with the tRNA ${ }^{\mathrm{Ser}(\mathrm{UCN})} / \mathrm{CO} 1 \mathrm{G} 7444 \mathrm{~A}$ mutation. ${ }^{41}$

Several nuclear genes have been associated with phenotypic modulation of the A1555G mtDNA mutation. The first identified modifier gene TFB1M has been shown to be directly involved in mitochondrial $12 \mathrm{~S}$ rRNA methylation. ${ }^{42,43}$ MTO1 and GTPBP3 were proposed to be part of the mitochondrial tRNA modification machinery. ${ }^{44,45}$ Another study ${ }^{46}$ identified the nuclear modifier gene TRMU for the phenotypic expression of deafness-associated mitochondrial $12 \mathrm{~S}$ rRNA mutations. A point mutation (A10S) in TRMU results in the decrease of the steady-state level of mt tRNAs, subsequently causing the impairment of mitochondrial translation. Resultant biochemical defects aggravate the mitochondrial dysfunction below the threshold for normal cell function, thereby expressing the deafness phenotype. Also, it cannot be excluded that human mitochondrial genes have functions in addition to their functions in oxidative phosphorylation. In this model, the mitochondrial mutation would interfere with a tissue-specific secondary function of the mitochondrial gene, which also has to make the cell more sensitive to changes in oxidative phosphorylation capacity. ${ }^{36}$

Another significant factor that determines the expression of mitochondrial disease is heteroplasmy. The A1555G mtDNA mutation had been thought to transmit only in a homoplasmic state, but heteroplasmic cases have also been found to exist and furthermore to be associated with severe deafness. ${ }^{47}$ Analysis of genotype-phenotype correlation indicated that subjects carrying $<20 \%$ of mutant copies were asymptomatic or had a mild hearing loss. ${ }^{47}$ However, it should be noted that it is difficult to determine the correlation of heteroplasmy levels with severity of hearing loss because the mutation load in blood may be different from that occurring in the inner ear. ${ }^{48}$

It has been suggested ${ }^{34}$ that a synergism between GJB2 and A1555G mutations may be possible and can be explained by an ability to maintain the normal turnover rate of the gap junction protein Cx26 because of the reduced amount of ATP caused by the A1555G mutation. Mitochondrial mutations that adversely affect the cell's ability to efficiently utilize metabolites for energy production may disrupt cochlear cells from maintaining needed levels of gap junctions. Even if the cell slowed the turnover of connexins in response to the lower energy state, the cell would lose the adaptability provided by the continuous remodeling of gap junction channels. ${ }^{34}$ Indeed, the A1555G or the C1494T mtDNA mutation produces a $\sim 30$ or 
$\sim 40 \%$ decrease in the rate of mitochondrial translation, respectively. ${ }^{7,49,50}$ However, a $\sim 50 \%$ decrease in the rate of mitochondrial translation responsible for significant respiratory defects was proposed as the threshold level that produces the deafness phenotype associated with the A1555G or the C1494T mutations. ${ }^{49,50}$

After summarizing data from the families reported to harbor both an mtDNA deafness-causing mutation and a GJB2 mutation (Table 1), conclusions might seem contradictory and confusing. The four patients of the Spanish family harboring the A1555G mtDNA mutation and the 35delG GJB2 mutation, were not subjected to aminoglycosides and had a moderate, postlingual hearing loss with an age of onset at $16-30$ years $^{17}$ (I-IV, Table 1). It is interesting to note that two members of this family, with the A1555G but not the $35 \mathrm{delG}$ mutation, also presented with moderate postlingual hearing impairment (V and VI, Table 1). In the described Japanese family, ${ }^{34}$ three siblings with the A1555G mutation were subjected to aminoglycosides (VII-IX, Table 1). One of them also had the 235delC GJB2 mutation (IX, Table 1). All three presented with late-onset progressive hearing loss but the sibling with the $235 \mathrm{delC}$ mutation had profound deafness (Table 1). In the first Greek family, ${ }^{35}$ the proband (X, Table 1) and his brother (XI, Table 1) had the A1555G mtDNA and the 35delG GJB2 mutations. Their mother had the A1555G mtDNA but not the 35delG GJB2 mutation. The proband and his mother had received aminoglycosides; however, although the proband had a moderate prelingual hearing loss with an age of onset at 5 months, his mother had normal hearing. Remarkably, his brother, with the A1555G and the 35delG mutations, but no exposure to aminoglycosides, presented with a moderate postlingual hearing impairment (XI, Table 1). It is interesting to note that in the second Greek family, ${ }^{35}$ the proband with the A1555G but not the 35delG mutation, and also subjected to aminoglycosides, had a profound postlingual deafness, with an age of onset at 2 years (XII, Table 1). His three male siblings, also with the A1555G mutation, had normal hearing. ${ }^{35}$

Interpreting data from the Spanish family, one might conclude that the 35delG GJB2 mutation does not seem to modify the deafness phenotype due to the A1555G mtDNA mutation. On the other hand, data from the Japanese family imply that there is indeed a synergism between the A1555G mtDNA and 235delC GJB2 mutations. Interpretation of data becomes even more confusing when comparing the two families with the A1555G mtDNA mutation from Greece. In the first family, aminoglycosides did not appear to have any impact on the mother with the A1555G, but did affect the proband which also had the $35 \mathrm{delG}$ mutation (X, Table 1). Strikingly, the proband (X, Table 1), which was exposed to aminoglycosides, had the same degree of hearing loss with his brother (XI, Table 1) who had the same two mutations but no history of ototoxic drugs. If we accept the assumption that GJB2 mutations can be an aggravating factor to the phenotypic expression of the A1555G mtDNA mutation, then the proband of the second family, with the A1555G and aminoglycoside exposure but not the $35 \mathrm{delG}$ mutation (XII, Table 1), should have presented with hearing loss less severe than the proband of the first family (X, Table 1), which is not the case.

So far, it is well-documented that aminoglycosides are ototoxic drugs that can not only trigger hearing impairment in individuals with mtDNA mutations but also in persons 'without' mtDNA deafnesscausing mutations. In addition, although the carrier frequency of GJB2 mutations, and especially the carrier frequency of the GJB2 35delG mutation, has been determined in several populations worldwide, it is known that the frequency of GJB2 heterozygotes among deaf patients in a population can be higher than the determined carrier frequency in this population. This fact suggests that there might be other genetic or environmental factors that modulate the expression of deafness in individuals with only one GJB2 mutant allele. To date, the experience gained in the field of mtDNA deafness-causing mutations, and especially the A1555G mutation, demonstrates that several factors participate as either additive or synergistic, but definitely modifying the phenotypic expression. The approach of candidate genes is a strategy that complements the screening approach. Mitochondrial rRNA and tRNA modification is likely to have an important role in determining the phenotype of non-syndromic hearing loss and perhaps mitochondrial disorders in general. More studies are needed to shed light on the multifactorial complex of the phenotypic expression of deafness-causing mtDNA mutations.

\section{ACKNOWLEDGEMENTS}

This study was supported in part by a grant from Oticon Fonden, Denmark (MBP).

1 Davis, A. C. The prevalence of hearing impairment and reported hearing disability among adults in Great Britain. Int. J. Epidemiol. 18, 911-917 (1989).

2 Marazita, M. L., Ploughman, L. M., Rawlings, B., Remington, E., Arnos, K. S. \& Nance, W. E. Genetic epidemiological studies of early-onset deafness in the U.S. school-age population. Am. J. Med. Genet. 46, 486-491 (1993).

3 Jacobs, H. T., Hutchin, T. P., Käppi, T., Gillies, G., Minkkinen, K., Walker, J. et al. Mitochondrial DNA mutations in patients with postlingual, nonsyndromic hearing impairment. Eur. J. Hum. Genet. 13, 26-33 (2005).

4 Giles, R. E., Blanc, H., Cann, H. M. \& Wallace, D. C. Maternal inheritance of human mitochondrial DNA. Proc. Natl Acad. Sci. USA. 77, 6715-6719 (1980).

5 Dimauro, S. \& Davidzon, G. Mitochondrial DNA and disease. Ann. Med. 37, 222-232 (2005).

6 Sudoyo, H., Suryadi, H., Lertrit, P., Pramoonjago, P. Lyrawati, D. \& Marzuki, S. Asianspecific mtDNA backgrounds associated with the primary G11778A mutation of Leber's hereditary optic neuropathy. J. Hum. Genet. 47, 594-604 (2002).

7 Guan, M. X., Fischel-Ghodsian, N. \& Attardi, G. Nuclear background determines biochemical phenotype in the deafness-associated mitochondrial 12S rRNA mutation. Hum. Mol. Genet. 10, 573-580 (2001).

8 Kokotas, H., Petersen, M. B. \& Willems, P. J. Mitochondrial deafness. Clin. Genet. 71, 379-391 (2007).

9 Linnane, A. W., Marzuki, S., Ozawa, T. \& Tanaka, M. Mitochondrial DNA mutations as an important contributor to ageing and degenerative diseases. Lancet 1, 642-645 (1989).

10 de Grey, A. D. Reactive oxygen species production in the mitochondrial matrix: implications for the mechanism of mitochondrial mutation accumulation. Rejuvenation Res. 8, 13-17 (2005).

11 Estivill, X., Govea, N., Barceló, E., Badenas, C., Romero, E., Moral, L. et al. Familial progressive sensorineural deafness is mainly due to the mtDNA A1555G mutation and is enhanced by treatment of aminoglycosides. Am. J. Hum. Genet. 62, 27-35 (1998).

12 Li, R., Xing, G., Yan, M., Cao, X., Liu, X. Z., Bu, X. et al. Cosegregation of C-insertion at position 961 with the $\mathrm{A} 1555 \mathrm{G}$ mutation of the mitochondrial $12 \mathrm{~S}$ rRNA gene in a large Chinese family with maternally inherited hearing loss. Am. J. Med. Genet. A 124, 113-117 (2004).

13 Matthijs, G., Claes, S., Longo-Mbenza, B. \& Cassiman, J. J. Non-syndromic deafness associated with a mutation and a polymorphism in the mitochondrial $12 \mathrm{~S}$ ribosomal RNA gene in a large Zairean pedigree. Eur. J. Hum. Genet. 4, 46-51 (1996).

14 Prezant, T. R., Agapian, J. V., Bohlman, M. C., Bu, X., Oztas, S., Qiu, W. Q. et al. Mitochondrial ribosomal RNA mutation associated with both antibiotic-induced and non-syndromic deafness. Nat. Genet. 4, 289-294 (1993).

15 Zhao, H., Li, R., Wang, Q., Yan, Q., Deng, J. H., Han, D. et al. Maternally inherited aminoglycoside-induced and nonsyndromic deafness is associated with the novel C1494T mutation in the mitochondrial 12S rRNA gene in a large Chinese family. Am. J. Hum. Genet. 74, 139-152 (2004).

16 Pandya, A., Xia, X., Radnaabazar, J., Batsuuri, J., Dangaansuren, B., Fischel-Ghodsian, $\mathrm{N}$. et al. Mutation in the mitochondrial 12S rRNA gene in two families from Mongolia with matrilineal aminoglycoside ototoxicity. J. Med. Genet. 34, 169-172 (1997).

17 López-Bigas, N., Rabionet, R., Martinez, E., Bravo, O., Girons, J., Borragan, A. et al. Mutations in the mitochondrial tRNA Ser(UCN) and in the GJB2 (connexin 26) gene are not modifiers of the age at onset or severity of hearing loss in Spanish patients with the 12S rRNA A1555G mutation. Am. J. Hum. Genet. 66, 1465-1467 (2000).

18 Chu, S. Y., Chiang, S. C., Chien, Y. H. \& Hwu, W. L. Screening of mitochondrial DNA mutations in subjects with non-syndromic familial hearing impairment in Taiwan. Acta. Paediatr. Taiwan 43, 330-333 (2002).

19 Yuan, H., Qian, Y., Xu, Y., Cao, J., Bai, L., Shen, W. et al. Cosegregation of the G7444A mutation in the mitochondrial COI/tRNA(Ser(UCN)) genes with the 12S rRNA A1555G mutation in a Chinese family with aminoglycoside-induced and nonsyndromic hearing loss. Am. J. Med. Genet. 138, 133-140 (2005). 
20 Zhu, Y., Qian, Y., Tang, X., Wang, J., Yang, L., Liao, Z. et al. Aminoglycoside-induced and non-syndromic hearing loss is associated with the G7444A mutation in the mitochondrial COI/tRNASer(UCN) genes in two Chinese families. Biochem. Biophys. Res. Commun. 342, 843-850 (2006).

21 Abreu-Silva, R. S., Lezirovitz, K., Braga, M. C., Spinelli, M., Pirana, S., Della-Rosa, V. A. et al. Prevalence of the A1555G (12S rRNA) and tRNASer(UCN) mitochondrial mutations in hearing-impaired Brazilian patients. Braz. J. Med. Biol. Res. 39, 219-226 (2006).

22 Yuan, H., Chen, J., Liu, X., Cheng, J., Wang, X., Yang, L. et al. Coexistence of mitochondrial 12S rRNA C1494T and C01/tRNA(Ser(UCN)) G7444A mutations in two Han Chinese pedigrees with aminoglycoside-induced and non-syndromic hearing loss. Biochem. Biophys. Res. Commun. 362, 94-100 (2007).

23 Jin, L., Yang, A., Zhu, Y., Zhao, J., Wang, X., Yang, L. et al. Mitochondrial tRNASer (UCN) gene is the hot spot for mutations associated with aminoglycoside-induced and non-syndromic hearing loss. Biochem. Biophys. Res. Commun. 361, 133-139 (2007).

24 Rydzanicz, M., Wróbel, M., Cywiñska, K., Froehlich, D., Gawecki, W., Szyfter, W. et al. Screening of the general Polish population for deafness-associated mutations in mitochondrial 12S rRNA and tRNA Ser(UCN) genes. Genet. Test Mol. Biomarkers 13, 167-172 (2009).

25 Hernandez, V. H., Bortolozzi, M., Pertegato, V., Beltramello, M., Giarin, M., Zaccolo, M. et al. Unitary permeability of gap junction channels to second messengers measured by FRET microscopy. Nat. Methods 4, 353-358 (2007).

26 Rabionet, R., Gasparini, P. \& Estivill, X. Molecular genetics of hearing impairment due to mutations in gap junction genes encoding beta connexins. Hum. Mutat. 16, 190202 (2000).

27 Richard, G., Rouan, F., Willoughby, C. E., Brown, N., Chung, P., Ryynänen, M. et al. Missense mutations in GJB2 encoding connexin-26 cause the ectodermal dysplasia keratitis-ichthyosis-deafness syndrome. Am. J. Hum. Genet. 70, 1341-1348 (2002).

28 Ballana, E., Ventayol, M., Rabionet, R., Gasparini, P., Estivill, X. Connexins and deafness homepage, http://davinci.crg.es/deafness/.

29 Petersen, M. B. \& Willems, P. J. Non-syndromic, autosomal-recessive deafness. Clin. Genet. 69, 371-392 (2006).

30 Denoyelle, F., Marlin, S., Weil, D., Moatti, L., Chauvin, P., Garabédian, E. N. et al. Clinical features of the prevalent form of childhood deafness, DFNB1, due to a connexin-26 gene defect: implications for genetic counselling. Lancet 353, 1298-1303 (1999).

31 Cohn, E. S., Kelley, P. M., Fowler, T. W., Gorga, M. P., Lefkowitz, D. M., Kuehn, H. J. et al. Clinical studies of families with hearing loss attributable to mutations in the connexin 26 gene (GJB2/DFNB1). Pediatrics 103, 546-550 (1999).

32 Kokotas, H., Theodosiou, M., Korres, G., Grigoriadou, M., Ferekidou, E., GiannouliaKarantana, A. et al. Sudden hearing loss in a family with GJB2 related progressive deafness. Int. J. Pediatr. Otorhinolaryngol. 72, 1735-1740 (2008).

33 Torroni, A., Cruciani, F., Rengo, C., Sellitto, D., López-Bigas, N., Rabionet, R. et al. The A1555G mutation in the 12S rRNA gene of human mtDNA: recurrent origins and founder events in families affected by sensorineural deafness. Am. J. Hum. Genet. 65, 1349-1358 (1999).

34 Abe, S., Kelley, P. M., Kimberling, W. J. \& Usami, S. I. Connexin 26 gene (GJB2) mutation modulates the severity of hearing loss associated with the $1555 \mathrm{~A} \rightarrow \mathrm{G}$ mitochondrial mutation. Am. J. Med. Genet. 103, 334-338 (2001).
35 Kokotas, H., Grigoriadou, M., Korres, G. S., Ferekidou, E., Papadopoulou, E., Neou, P. et al. The A1555G mitochondrial DNA mutation in Greek patients with non-syndromic, sensorineural hearing loss. Biochem. Biophys. Res. Commun. 390, 755-757 (2009).

36 Fischel-Ghodsian, N. Mitochondrial mutations and hearing loss: paradigm for mitochondrial genetics. Am. J. Hum. Genet. 62, 15-19 (1998).

37 Brummett, R. E., Fox, K. E., Bendrick, T. W. \& Himes, D. L. Ototoxicity of tobramycin, gentamicin, amikacin and sisomicin in the guinea pig. J. Antimicrob. Chemother. 4 (Suppl.), 73-83 (1978).

38 Brummett, R. E. \& Morrison, R. B. The incidence of aminoglycoside antibiotic-induced hearing loss. Arch. Otolaryngol. Head Neck Surg. 116, 406-410 (1990).

39 Schacht, J. Biochemical basis of aminoglycoside ototoxicity. Otolaryngol. Clin. North Am. 26, 845-856 (1993).

$40 \mathrm{Lim}, \mathrm{D}$. J. Effects of noise and ototoxic drugs at the cellular level in the cochlea: a review. Am. J. Otolaryngol. 7, 73-99 (1986).

41 Lu, J., Qian, Y., Li, Z., Yang, A., Zhu, Y., Li, R. et al. Mitochondrial haplotypes may modulate the phenotypic manifestation of the deafness-associated $12 \mathrm{~S}$ rRNA 1555A > G mutation. Mitochondrion 10, 69-81 (2010).

42 Bykhovskaya, Y., Mengesha, E., Wang, D., Yang, H., Estivill, X., Shohat, M. et al. Human mitochondrial transcription factor B1 as a modifier gene for hearing loss associated with the mitochondrial A1555G mutation. Mol. Genet. Metab. 82, 27-32 (2004).

43 Seidel-Rogol, B. L., McCulloch, V. \& Shadel, G. S. Human mitochondrial transcription factor B1 methylates ribosomal RNA at a conserved stem-loop. Nat. Genet. 33, 23-24 (2003).

$44 \mathrm{Li}, \mathrm{X}$. Li, R., Lin, X. \& Guan, M. X. Isolation and characterization of the putative nuclear modifier gene MTO1 involved in the pathogenesis of deafness-associated mitochondrial 12 S rRNA A1555G mutation. J. Biol. Chem. 277, 27256-27264 (2002).

$45 \mathrm{Li}$, X. \& Guan, M. X. A human mitochondrial GTP binding protein related to tRNA modification may modulate phenotypic expression of the deafness-associated mitochondrial 12S rRNA mutation. Mol. Cell Biol. 22, 7701-7711 (2002).

46 Guan, M. X., Yan, Q., Li, X., Bykhovskaya, Y., Gallo-Teran, J., Hajek, P. et al. Mutation in TRMU related to transfer RNA modification modulates the phenotypic expression of the deafness-associated mitochondrial $12 \mathrm{~S}$ ribosomal RNA mutations. Am. J. Hum. Genet. 79, 291-302 (2006).

47 del Castillo, F. J., Rodríguez-Ballesteros, M., Martín, Y., Arellano, B., Gallo-Terán, J., Morales-Angulo, C. et al. Heteroplasmy for the 1555A $>$ G mutation in the mitochondrial 12S rRNA gene in six Spanish families with non-syndromic hearing loss. J. Med. Genet. 40, 632-636 (2003).

48 Lu, S. Y., Nishio, S., Tsukada, K., Oguchi, T., Kobayashi, K., Abe, S. et al. Factors that affect hearing level in individuals with the mitochondrial 1555A>G mutation. Clin. Genet. 75, 480-484 (2009).

49 Guan, M. X., Fischel-Ghodsian, N. \& Attardi, G. Biochemical evidence for nuclear gene involvement in phenotype of non-syndromic deafness associated with mitochondrial 12S rRNA mutation. Hum. Mol. Genet. 5, 963-971 (1996).

50 Zhao, H. Young, W. Y. Yan, Q. Li, R. Cao, J. Wang, Q et al. Functional characterization of the mitochondrial 12S rRNA C1494T mutation associated with aminoglycoside-induced and non-syndromic hearing loss. Nucleic Acids Res. 33, 1132-1139 (2005) 